\title{
Índice de autores
}

A

\begin{tabular}{|c|c|c|c|c|c|}
\hline Alende Castro V & 87 & Fachal Bermúdez C & 89 & Machado B & 99 \\
\hline Alonso M & 92,105 & Fariña Sarasqueta S & 91,92 & Macía Rodríguez C & 87 \\
\hline Alonso Mesonero D & 86 & Fernández Bouza E & $90,93,100,103,104$ & Marcos P & 99 \\
\hline Alonso Parada M & 94,99 & Femández Fernández FJ & $89,92,95,96,97,101,103,104$ & Marey López J & 96 \\
\hline Álvarez Asensio E & 91,92 & & 105 & Mariño Callejo Al & 96,102 \\
\hline Álvarez Díaz H & 92 & Fernández González A & 89,100 & Martínez A & 87,105 \\
\hline Álvarez-Fernández M & 105 & Fernández I & 96 & Martínez Braña L & $88,91,95$ \\
\hline Álvarez J & 87,93 & Fernández J & 99 & Martínez-Lamas L & $87,92,105$ \\
\hline Álvarez Otero J & $89,91,92,96,98,101,103,104$ & Fernández JC & 102 & Martínez M & 95 \\
\hline & 105 & $\begin{array}{l}\text { Fernández Pérez C } \\
\text { Fernández Regal I }\end{array}$ & $\begin{array}{l}104 \\
86,94,97,101,104\end{array}$ & $\begin{array}{l}\text { Martínez Velado E } \\
\text { Martínez Vidal A }\end{array}$ & $\begin{array}{l}91,92 \\
94,97,100,103\end{array}$ \\
\hline Alves Pereira DE & 100 & & 88 & & $94,97,100,103$ \\
\hline Anibarro García L & $88,89,94,100$ & & $\begin{array}{l}\infty 0 \\
101\end{array}$ & $\begin{array}{l}\text { Mateo Mlosquera L } \\
\text { Matesanz M }\end{array}$ & $87,91,95$ \\
\hline Antela A & 91,95 & $\begin{array}{l}\text { Fernández Rodriguez } \mathrm{R} \\
\text { Fernández Rodríquez } \mathrm{V}\end{array}$ & 90,93 & Matesanz IM & 89,102 \\
\hline Aparicio M & 100 & & 90,93 & $\begin{array}{l}\text { Matute G } \\
\text { Meiiide H }\end{array}$ & $\begin{array}{l}95 \\
86889899101\end{array}$ \\
\hline Araújo Fernández S & $91,95,96,103,105$ & Fernández Somoza JM & 87,88 & $\begin{array}{l}\text { Melljde H } \\
\text { Mella Pérez C }\end{array}$ & $\begin{array}{l}86,88,98,99,101 \\
90,93,100,103,104\end{array}$ \\
\hline Arca A & 87 & Fernández Villaverde A & 96 & Mena A & $\begin{array}{l}90,93,100,103,104 \\
86,88,101\end{array}$ \\
\hline Arévalo A & 93 & Ferreira González L & 102 & Míguez Rey E & 86 \\
\hline $\begin{array}{l}\text { Arevalo Gomez A } \\
\text { Argibay A }\end{array}$ & $\begin{array}{l}86 \\
87,92,105\end{array}$ & Figueroa López RM & 89 & Molinos-Castro S & 91,102 \\
\hline Argibay Filgueira $A B$ & $92,94,97,100,103$ & Freire Castro S & 86 & Monteagudo Sánchez B & $91,92,104$ \\
\hline Arrojo Alonso F & 89,100 & Freire Dapena M & $94,95,99,103$ & Monte Secades R & 88 \\
\hline Asensio P & 98,99 & Freire S & 93 & $\begin{array}{l}\text { Montes J } \\
\text { Montes-Santiago J }\end{array}$ & $\begin{array}{l}99,103 \\
104\end{array}$ \\
\hline $\mathbf{B}$ & & G & & Moreno JA & 98,99 \\
\hline Bal Alvaredo M & 88 & García Alén D & $89,90,91,92,93,96,97,98,100$ & Moreno-Lugris C & 94 \\
\hline Balo-Araujo S & 91,102 & García García JC & $102,103,104$ & Mosquera Martínez I & 91 \\
\hline $\begin{array}{l}\text { Barbagelata López C } \\
\text { Baroja A }\end{array}$ & $86,93,100,104$ & García J & $\begin{array}{l}89,100 \\
100\end{array}$ & $\mathbf{N}$ & \\
\hline Baroja Basanta A & $\begin{array}{l}92,105 \\
94,99,103\end{array}$ & García Jiménez A & 102 & Naval Calviño G & 101 \\
\hline Beceiro Abad MC & $\begin{array}{l}94,99,100 \\
95\end{array}$ & García-País Mª J & 86 & Naveiro-Soneira J & 91,102 \\
\hline Bermúdez JR & 87 & García Poutón N & $89,92,96$ & Nicolás R & 88,104 \\
\hline Bermúdez Ramos M & $87,88,91$ & García-Suárez F & 91,102 & Nodar A & $87,92,105$ \\
\hline Boado Martínez M & 91,92 & Gayol-Fernández MC & 102 & Nodar Germiñas A & $92,97,100$ \\
\hline Bravo Blázquez I & $89,90,91,92,93,96,97,98,100$ & Gómez Buela I & $89,90,91,92,93,96,97,98,100$ & Novoa Lamazares L & 95 \\
\hline Bravo-Ferrer Acosta JM & $\begin{array}{l}102,104 \\
86\end{array}$ & Gómez Canosa S & 98 & Núñez Fernández MJ & 89,100 \\
\hline Brea Aparicio R & $89,94,100$ & Gómez Fernández R & 86 & Núñez MJ & 94 \\
\hline Bustillo Casado M & 101 & Gómez Gigirey A & 91 & & \\
\hline Busto E & 97,100 & Gómez JM & 87,92 & $\begin{array}{l}\text { O } \\
\text { Orona Nava M }\end{array}$ & 102 \\
\hline C & & $\begin{array}{l}\text { Gómez Sousa J } \\
\text { Gómez Sousa JM }\end{array}$ & $\begin{array}{l}103,105 \\
92,94,99,100\end{array}$ & Otero Alvarín E & 91,92 \\
\hline Caínzos Romero T & $89,90,91,92,96,97,98,100$ & Gómez-Tato C & 103 & Otero Antón E & 87,88 \\
\hline & $102,103,104$ & Gondar Barcala T & $89,92,96,98,101$ & Otero González I & 98 \\
\hline Calviño L & 94 & González A & 96 & $\mathbf{P}$ & \\
\hline Camba Estévez M & 105 & González Fernández C & 101 & Padín Paz EM & 91,102 \\
\hline Campos Franco J & 87,88 & González González L & $87,91,93,98,104$ & Pallarés A & 94 \\
\hline Carballo Fernández I & 95 & González Lorenzo JR & 97 & Pardo Souto MC & 98 \\
\hline Carrero J & 98,99 & González Noya A & $86,94,97,101,104$ & Pastor Rubín E & 102 \\
\hline Casariego $\mathrm{E}$ & 89,102 & González Quintela A & $87,88,95$ & Pato A & 93 \\
\hline Castelo-Corral LM & 86 & González Rodríguez M & 93 & Paz Ferrín J & 104 \\
\hline Castro A & 86,88 & González V & 98,99 & Pedreira JD & $86,88,101$ \\
\hline Castro-Iglesias A & 101 & González Vázquez L & $\begin{array}{l}97,101 \\
95\end{array}$ & Penado S & 88 \\
\hline Cidre R & 105 & Gozález-Rivero C & 95 & Penado V & 104 \\
\hline Clavero E & $97,99,100$ & Guillén C & 95 & PérezA & 99,103 \\
\hline $\begin{array}{l}\text { Clavero Fernandez E } \\
\text { Codesal Norniella A }\end{array}$ & $\begin{array}{l}96,98 \\
88\end{array}$ & I & & Pérez Carral 0 & 101,104 \\
\hline Coira A & 86,94 & Iglesias Gallego M & 91,102 & Pérez Carral V & $86,94,97,101,104$ \\
\hline Corredoira J & 86 & Îñiguez I & 89,102 & Pérez del Molino ML & 91,102 \\
\hline Criilo I & 93 & Î́niguez Vázquez I & 90 & Pérez R & 103 \\
\hline Crillo Calderón I & 104 & Isorna MJ & 88 & Pérez Rodríguez MT & $87,92,94,100,103,105$ \\
\hline & & Isusi Moure A & 86 & Pérez V & 96 \\
\hline & & & & Pérez Valcárcel J & 92 \\
\hline De la Fuente Aguado J & $87,89,91,92,93,95,96,97,98$, & Júnez & & Pernas B & $86,88,101$ \\
\hline & $101,103,104,105$ & Jimenez JL & 96 & Pesquelra-Fontan PIV & 91,102 \\
\hline De la lglesia F & 88,104 & Jimenez IVlartinez JL & 104 & $\begin{array}{l}\text { PIneiro Fernandez JC } \\
\text { Piñeiro J }\end{array}$ & $\begin{array}{l}90,93,94 \\
102\end{array}$ \\
\hline De Toro M & 97 & $\mathbf{L}$ & & Piñeiro Parga P & $\begin{array}{l}102 \\
86,91\end{array}$ \\
\hline De Toro Santos JM & 104 & Lamas Ferreiro JL & $87,91,93,98,101,104$ & Pombo B & 102 \\
\hline Díaz López MD & 97,101 & Lijó Carballeda C & $89,90,91,92,93,96,97,98,100$ & Porteiro J & 98,99 \\
\hline $\begin{array}{l}\text { Ulaz Peromingo JA } \\
\text { Diéguez Paz J }\end{array}$ & $\begin{array}{l}91,95,102 \\
89,100\end{array}$ & & $102,103,104$ & Porto AB & 88 \\
\hline Domínguez Rodríguez T & $\begin{array}{l}89,100 \\
86\end{array}$ & Llinares D & 93 & Porto Pérez AB & 86,91 \\
\hline Domínguez T & $\begin{array}{l}86 \\
88\end{array}$ & Llinares García D & 86 & Poveda E & $86,88,101$ \\
\hline Dubois A & 88,104 & Llinares Modéjar $\mathrm{P}$ & 86 & Puerta Louro R & $91,92,101$ \\
\hline Durán Muñoz D & 89 & $\begin{array}{l}\text { López-Alvarez Ma J } \\
\text { López Castro J }\end{array}$ & 86 & & \\
\hline E & & López J & 99 & & \\
\hline Echarri Piudo A & 97 & López M & 102 & & \\
\hline Edesio Fírvida J & 91,92 & López Mato A & 101 & & \\
\hline Enríquez de Salamanca | & $\begin{array}{l}1,92 \\
87\end{array}$ & López Mato P & $86,94,97,101,104$ & & \\
\hline Enríquez Gómez H & $95,96,103,105$ & López Mouriño VM & 91,92 & & \\
\hline Estévez M & 103 & López P & 96 & & \\
\hline Estéz M & 99 & López Reboiro ML & $86,88,90,93,94,102$ & & \\
\hline & & López S & $86,88,101$ & & \\
\hline & & López Vázquez M & 100 & & \\
\hline & & Lorenzo Castro R & $92,97,99,100,103$ & & \\
\hline & & Lorenzo R & 92,105 & & \\
\hline & & Losada E & 91,95 & & \\
\hline
\end{tabular}

$\mathbf{R}$

$\begin{array}{ll}\text { Rabuñal Rey R } & 86,90,93 \\ \text { Ramos V } & 88,104 \\ \text { García Alén D } & 100 \\ \text { Rial Rama MP } & 91,102 \\ \text { Rico P } & 98,99 \\ \text { Ríos Prego M } & 88,100 \\ \text { Rivera A } & 87,92,105 \\ \text { Rivera Gallego A } & 92,94,97,99,100,103 \\ \text { Rivera S } & 93 \\ \text { Rodil V } & 103 \\ \text { Rodríguez A } & 87,93 \\ \text { Rodríguez Álvarez AP } & 104 \\ \text { Rodríguez Álvarez P } & 86,94,97 \\ \text { Rodríguez Arias M } & 89,97,101 \\ \text { Rodríguez Cordero M } & 95 \\ \text { Rodríguez-Fernández S } 91,102 \\ \text { Rodríguez-Framil M } & 91,102 \\ \text { Rodríguez Gómez A } & 94,99,103,105 \\ \text { Rodríguez Gómez AM } & 97,100 \\ \text { Rodríguez González A } & 86 \\ \text { Rodríguez I } & 87 \\ \text { Rodríguez-Macia A } & 86 \\ \text { Rodríguez Mayo M } & 86 \\ \text { Rodriguez-Osorio I } & 86,88,101 \\ \text { Rodríguez P } & 96 \\ \text { Rodríguez Pérez MT } & 97 \\ \text { Rodriguez-Segade S } & 99 \\ \text { Rodríguez Vilela A } & 103 \\ \text { Romay E } & 89,102 \\ \text { Romay Lema E } & 90,93,94 \\ \text { Rubal Bran D } & 88,90 \\ \text { Rubal D } & 89,102 \\ & \\ \text { S } & \end{array}$

s

Sánchez Conde P 103

Sánchez Pérez-Mel MC 88

Sánchez Sobrino P 88

Sánchez Trigo S $\quad 89,90,91,92,96,97,98,100$,

$102,103,104$

chez Vidal E $\quad 86$

Sanjurjo A 93

$\begin{array}{ll}\text { Sanjurjo Rivo A } & 98 \\ \text { Santos Armentía E } & 97,98\end{array}$

Sardina Ferreiro R $\quad 89,90,91,92,93,96,97,98,100$

Seco E -

Seoane B $\quad 88,104$

Serrano J $\quad 98,99$

Sesma Sánchez P $\quad 90,93,98,103$

Sopeña Pérez-Argüelles B 89

Soto A $\quad 105$

Soto Peleteiro A $\quad 87,92,94,99,100,103$

Sousa A $\quad 87,105$

Sousa Domínquez A $\quad 92,94,97,100,103$

Sousa Regueiro D $\quad 86,91$

Suárez Amor OM 90

Suárez Fuentetaja R $\quad 86,96,98$

Suárez Gill R $\quad 93$

Suárez R $\quad 97,100$

$\mathbf{T}$

Tomé S $\quad 87,88$

Trigas Ferrín M 102

v

Valcárcel García MA $\quad 87,95$

Val Domínguez N ～94,95,103

Valle Feijoo ML $\quad 95,97,10$

Val N 92,105

Varela García PM $\quad 91,102$

Vares González M $\quad 86$

Vares M 93

Varez González M $\quad 86$

Vázquez Ledo $\mathrm{L} \quad 87$

Vázquez $\mathrm{P} \quad 86,88,10$

Vázquez $\mathrm{R} \quad 88,104$

Vázquez Triñanes C $\quad 94,100,103$

Vázquez Vázquez B 93

Velo García A $\quad 88,89,94,100$

Ventura P $\quad 86,89,102$

Ventura Valcárcel P $\quad 88,90,93,94$

Verdeal Dacal R $\quad 86,96,98$

Verdeal R $\mathrm{g7,100}$

Vilariño Maneiro L $\quad 90,91,92,96,97,98,100,102$,

104

Villaverde Álvarez| $\quad$ 92,94,103 